\title{
Multicast Routing in Computer Networks considering Quality of Service (QoS) based on Honey Bee Algorithm
}

\author{
Mohamad Tayeb Taher, \\ Islamic Azad University, Zanjan branch, Zanjan, \\ Iran
}

\begin{abstract}
The main purpose of multicast routing is minimizing the sources relationship by using multicast structure and the purpose will prepare the routing by minimizing the tree's cost. The Steiner tree's issue is to find a tree the least cost in the network in a way that total weights of the tree should be minimized and connect one or more sources to one or more destinations. The weights which are applied to the edge of the tree could include one or more limitations of quality of service such as bandwidth, delay and cost. It is improved that finding such a tree in the network is a NP-COMPLETE problem. So, a real answer can't be find for that and approximate and discovering solutions are proposed for that. In this paper a suitable answer for mentioned NP-COMPLETE problem is found by presenting a new algorithm according to bee algorithm. Bee algorithm is a collective smart searching algorithm for optimizing the solution of problem. The applied results show that the proposed algorithm with regard to genetic algorithm and practice swarm optimization of Steiner tree with more suitable application percent computers the relation among bandwidth, delay and cost.
\end{abstract}

\section{General Terms}

multicast routing algorithm

\section{Keywords}

multicast routing, multicast tree, quality of service, Bee algorithm

\section{INTRODUCTION}

Nowadays, by developing information technology, the number of multimedia applied programs in network has been developed and it is developing. The examples of them which are videocast, teleconference, electronic education and etc. need multicast routing with assured service quality. There are three mechanism of addressing unicast, broadcast and multicast to send data in internet. Multicast connection can be applied in several ways. One possible method is that each send use a private unicast connection for every receiver. Another method is based on that network layer should protect the act of multicasting in a way that routers repeat the data on output routs where ever required according to routing able to send the data to all of the group members. This method uses more effective network bandwidth. The purpose of multicast routing if to find a tree which should include all routers have connected and related hosts to the group. Multicast data among the tree will be routing from the sender to all related hosts to the tree. Of course, the routings that don't have any host related to the group can be in tree structure. Steiner tree is a tree with the least cost in network that the total of all weights is minimized. The main advantage of the tree is economizing in network sources application. Finding such as

\author{
Amir Masoudrahmani, \\ Islamic Azad University, Science and Research \\ Branch, Tehran, Iran
}

tree in the network is a NP-COMPLETE issue. So a real answer for that can't be find and approximate and discovering solution is proposed for that. Several articles have been presented in this area.[1]The presented algorithm optimizes multicast tree directly. This algorithm uses PSO as a solution to control the tree frame optimization position.[2]The multicast routing algorithm with limitation of service quality is upon PSO algorithm that it first formulized the issue mathematically. According to PSO algorithm and then changed it to an algorithm.[3] First it modulated the multicast issue to the math then found on algorithmic definition according to some incorrect network parameters in considered algorithm that can find the limitations of service quality and according to the algorithm found an answer for NPCOMPLETE issue.[4]it proposes an ant algorithm for the issue. The defined algorithm has added the factor of determining the direction to the based algorithm of ant the makes the ant to get rid of primary blinding in time of searching the rout. This makes it possible to use results and reduces the function of losing the irrelevant routes. Therefore, it has come over the lack of the same direction in base ant algorithm.[5]It's an algorithm composed of ACO and PSO. Updating method of position in PSO in ACO production rule has been used in compound algorithm that the best position with the best solution of particles is the best solution of all updated particles. Furthermore, updating strategy in PSO was redefined by redefinition of mathematic operators.[6]It is genetic algorithm with the least coast and limitation of bandwidth and delaying that presents its genotype in the tree but no definitions of decoding and encoding are presented in this paper.[7]several genetic algorithm structure were considered and at last the genetic algorithm with two new operators was presented that had approximate results.

Bee algorithm has paid more attention in recent years and has shown a good productivity in the issue of optimization. Finding the best area was not worked as much in recent works and with this respects that bee algorithm has presented a good solution to get rid of solving the issue by using Bee algorithm. This fact includes five parts that in part two issues of multicast routing and service quality parameters were depicted in mathematic language. In part three, bee algorithm is explained. In part four relevant issue solutions were presented according to bee algorithm. In fifth the results of simulating and in sixth part the conclusion is mentioned.

\section{Multicast routing with considering the criteria of service quality base on bee algorithm}

We consider the network like a connected graph $\mathrm{G}=(\mathrm{V}, \mathrm{E})$ where $\mathbf{V}$ is the group of all centers (networks nodes) and $\mathbf{E}$ is the group of all edges (network links). Three real and not negative amount of $\mathbf{R}$ is related to each edge of $e \in E$. Cost 
function is $\mathrm{C}(\mathrm{e}): \mathrm{E} \rightarrow \mathrm{R}$, edge delaying function is $\mathrm{D}(\mathrm{e}): \mathrm{E} \rightarrow \mathrm{R}$, bandwidth function is $B(e): E \rightarrow R$.

$\mathrm{D}(\mathrm{e})$ and $\mathrm{B}$ (e) is functions that are as critical points in networks and have always limitation. A multicast tree with $\mathrm{T}$ (s, $\mathrm{m}$ ) shows that $\mathbf{S}$ is the root of multicast tree or on the other hand it's a sender in multicast group. $m=|\mathrm{M}|$ is the number of destination nodes in multicast tree. $\mathbf{M}$ is the group of destination nodes. Multicast tree is a member of graph $\mathbf{G}$ that covers source nod $s \in V$ and the group of destination node $\mathrm{M} \subseteq$ $\mathrm{V}-\{\mathrm{s}\}$.

\section{The definition of limitation of quality of service:}

service quality in the issue includes the parameters of bandwidth, coast and delaying.

The cost of multicast tree $\mathrm{T}(\mathrm{s}, \mathrm{M})$ is as total costs of tree edges and is computed as follow:

$\mathrm{C}(\mathrm{T}(\mathrm{S}, \mathrm{M}))=\sum_{e \in T(s, M)} C(e)$

The whole delay of the tree $\mathrm{T}(\mathrm{s}, \mathrm{m})$ is total delay of all edges of the tree $\mathrm{T}(\mathrm{s}, \mathrm{m})$ and is computed as follow:

$\mathrm{D}(\mathrm{T}(\mathrm{s}, \mathrm{M}))=\sum_{e \in T(s, M)} D(e)$

the whole bandwidth of the tree $\mathrm{T}(\mathrm{s}, \mathrm{m})$ is total bandwidth of the entire tree edges $\mathrm{T}(\mathrm{s}, \mathrm{m})$ and is computed as follow:

$\mathrm{B}(\mathrm{T}(\mathrm{s}, \mathrm{M}))=\sum_{e \in T(s, M)} B(e)$

limitation of bandwidth in the tree $\mathrm{T}(\mathrm{s}, \mathrm{m})$ as the least bandwidth available in the tree is defined as follow:

$\mathrm{B}(\mathrm{T}(\mathrm{s}, \mathrm{M}))=\operatorname{Min}\{\mathrm{B}(\mathrm{e}) \mid \mathrm{e} \in \mathrm{T}(\mathrm{s}, \mathrm{M})\}$

If $\Delta \mathrm{d}$ delay limitation and Bdbandwidth limitation are considered by us, the issue of multicast tree with delay and bandwidth limitation is defined as follow:

$$
\begin{aligned}
& \mathrm{D}(T(\mathrm{~s}, \mathrm{M})) \leq \Delta d \forall e \in T(s, M) \\
& \mathrm{B}(T(\mathrm{~s}, \mathrm{M})) \geq \mathrm{Bd} \quad \forall e \in T(s, M)
\end{aligned}
$$

All of edges which their bandwidths are lower than the least amount of need bandwidth of applying program were omitted. If both source node graph and destination nodes were not connected in a member graph, the bandwidth limitation would not be accomplished. So source node should go on an agreement with its applied program to improve the bandwidth otherwise the applied program will not be performable on this topology. The act is done on delaying too.

\section{The purpose of research}

The purpose of research is to achieve a multicast tree with appropriate fitness.

\section{Bee algorithm}

An optimization algorithm is based on population the makes pattern from the bee behavior to find the food. Like bee's behavior in nature, optimization algorithm is a group of bee with $\mathrm{X}$ pioneer bee that was placed randomly in searching area. In next step the fitness of data sites are evaluated by bees. Then Y bees that have the highest fitness are selected as chosen bees and sited were observed by them are selected to search the neighbor. Searching the neighbor for this purpose is used that it is possible that there are sites with higher fitness in the neighbor of selected sites' neighbor. To search the neighboring, the radius of neighborhood should be determined. We divide $\mathrm{Y}$ bees with higher fitness into two part $\mathrm{H}$ bees with the highest fitness and $\mathrm{Y}-\mathrm{H}$ bees with lower fitness.

Then some bees are made to work to search in selected neighborhood. For this purpose $\mathrm{X} 1$ bees to search around $\mathrm{h}$ site with higher fitness and $\mathrm{X} 2$ bees to search around $\mathrm{Y}-\mathrm{H}$ sites are used. Usually the number of bees around the site $\mathrm{H}$ is more than the number of bees for site $\mathrm{Y}-\mathrm{H}$.

In the next step each bee is evaluated and compared with bees as a neighbor near the area of neighborhood and if there is a neighbor with higher fitness, the neighbor bee will replace with the primary bee and the next population will be formed. $\mathrm{X}-\mathrm{Y}$ remained bee from the primary population is used to randomly searching in searching area and there are new site to replace for the next population. The steps of searching around randomly will repeat until the stop criterion is confirmed. The criteria of stop algorithm is achieving to the maximum repetition of algorithm to find the focus with the same direction for one answer. At the end of each repetition of the new population each colony includes two parts are: the best bees of each site with the number of $\mathrm{Y}$ and pioneer bees that has searched randomly with the number of X-Y. [8][9].

Providing initial population consist of $\mathrm{X}$ bee randomly

\section{Computing amount of fitness each member of population}

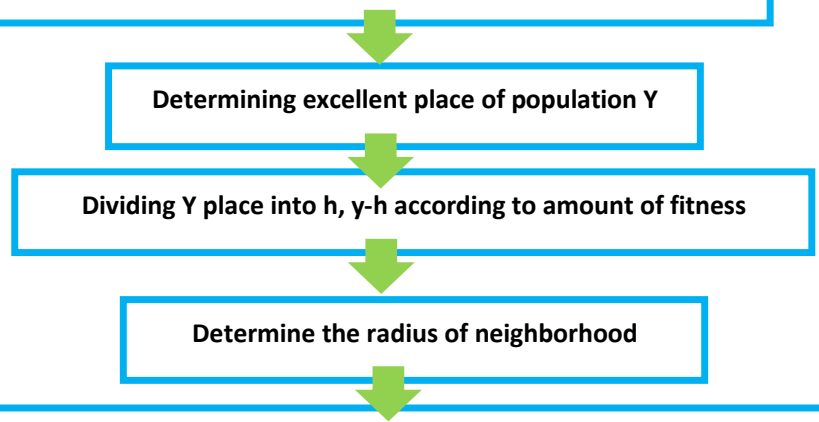

Sending more bee to radius of neighborhood according toamount of fitness

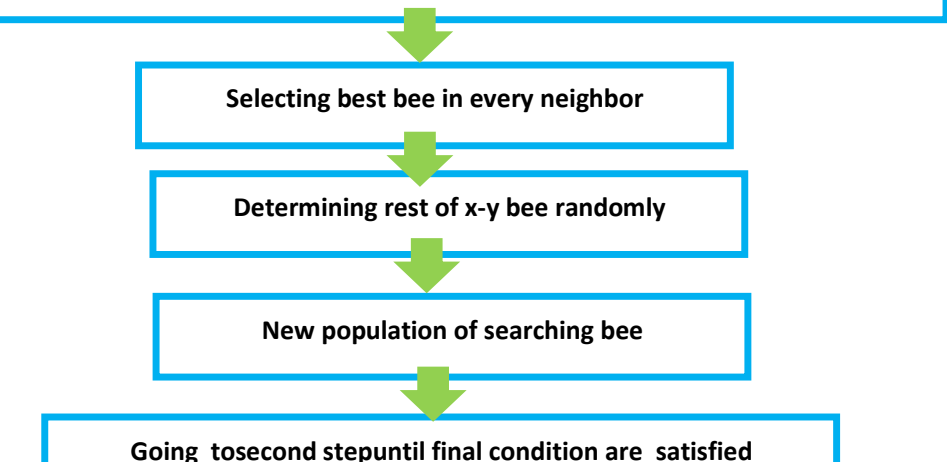

Going tosecond stepuntil final condition are satisfied 


\section{Solving the issue in multicast tree with bee algorithm}

The proposed algorithm includes 4 steps that are explained in detail.

\section{TheFirst step}

At first a number of trees which include relevant senders and receivers were created randomly. These trees are the primary population. We name each tree an especial routes or particle. After that the amount of fitness of each route was calculated by fitness function.

\section{The definition of fitness}

The new fitness function is used as a criteria and an amount to comparison among multicast trees. There is bandwidth if there was a reduction in the function in a way that the higher the multicast tree bandwidth the more suitable tree will have for the application program. There is cost and delay of the tree in the determination of fraction that the lower it is the more fitness will have. The ratio of the lowest bandwidth to the highest bandwidth causes the tree that has more balance in bandwidth difference has the higher fitness. The ratio among bandwidth in the tree to the highest bandwidth also causes that a tree which its bandwidth average has lower difference than the highest bandwidth will have the highest fitness.

$\mathrm{K}$ is the fitness efficient that has been considered according to the parameters of issue and the results of experiments.

$\operatorname{Fitness}(T(s, M))=$

$(1 / K) *\left(\frac{\frac{\mathrm{B}(\mathrm{T}(\mathrm{s}, \mathrm{M}))}{\mathrm{B}(\mathrm{G}(\mathrm{V}, \mathrm{e}))} * \frac{\text { Bmin }}{\text { Bmax }} * \frac{\text { Bavg }}{\text { Bmax }}}{\frac{\mathrm{C}(\mathrm{T}(\mathrm{S}, \mathrm{M}))}{\mathrm{C}(\mathrm{G}(\mathrm{V}, \mathrm{e}))}+\frac{\mathrm{D}(\mathrm{T}(\mathrm{s}, \mathrm{M}))}{\mathrm{D}(\mathrm{G}(\mathrm{V}, \mathrm{e}))}}\right)$

In this formula $\mathrm{T}(\mathrm{S}, \mathrm{M})$ is multicast tree, $\mathrm{B}(\mathrm{T}(\mathrm{S}, \mathrm{M}))$ bandwidth of multicast tree, $\mathrm{B}(\mathrm{G}(\mathrm{v}, \mathrm{e}))$ bandwidth of network Graph, Bmin, Minimum bandwidth in multicast tree, Bmax, maximum bandwidth in multicast tree, Bavg, the average of multicast tree bandwidth, $\mathrm{C}(\mathrm{T}(\mathrm{S}, \mathrm{M}))$ the cost in multicast tree, $\mathrm{C}(\mathrm{G}(\mathrm{v}, \mathrm{e}))$ the cost of network Graph, $\mathrm{D}(\mathrm{T}(\mathrm{S}, \mathrm{M}))$ delay in multicast tree and $\mathrm{D}(\mathrm{G}(\mathrm{v}, \mathrm{e}))$ the delay of network Graph.

Step 1

//each particle is tree include source and destination node

Determine number particle

For each particle

Build tree randomly

Delete node with degree one and not

destination

End for

Calculate fitness for each particle

Sort the particle based on fitness

\section{The two step}

All of the population was divided into the three parts, elites of first level and elites of second level and the remained routes, according to amount of fitness function that their each portion are $25 \%, 35 \%, 40 \%$ of all of the primary population respectively and approximately with the respect of experiments' results in the best conditions.
The acts relevant to the elites of first level are performed according to bee algorithm. At first a number of routes which are in the neighbor of elites' rout for each of elites were created then amount of fitness of routes of its neighbor is calculated and if there were neighbors with higher fitness, it would be the substitution of elite route.

Neighbor route is obtained by using elite route as in nodes there is difference in amount of radius of its elite's neighbor routes. Neighbor radius is the quantity percent of route nodes. Neighbor route includes elite route centers except several nodes that are omitted randomly. It means that the routes are made include centers of elite route except omitted centers.

Step 2

Determine number of elites level 1 and elites level 2

Determine number neighbor

For elites level 1 to elites level 2

For each neighbor

Delete some node form elite tree

Build multicast tree randomly that include vertex elite tree

Delete node with degree one and not destination

//Neighbor tree is different with elite tree in some node

End for (for line 2)

For each neighbor

Calculate the fitness of neighbor elite

If neighbor has better fitness

neighbor replace with Elite

End for (for line 7)

End for (for line 1)

The third step:

The steps of first level elites are exactly performed for elites of second level. With this difference that for each of the level elites, lower number of neighbors is considered and the level of main loop is also equal to the number of second level elites. In this level for each of elite routes a number of neighbors are also considered and if a neighbor with higher amounts of fitness is found, it will be its elite substitution.

Step 3

//This step is copy of step 1 only number neighbor in this step is more

//in this step index of cycle for is equal elites level 2

\section{The fourth step:}

At last, the remained routes of first population are replaced with routes created randomly. Then the amount of fitness of all new population routes is calculated and algorithm will be repeated in order to achieve the appropriate answer or to finish the number of repetition.

Step 4

For elites level2 to end of particle

Build new tree for each particle randomly

Calculate the new population use of step1,step2,step3 particle and go to step 1 


\section{Simulating and evaluating the algorithm}

$\mathrm{C} \neq$.Net programming language has been used to simulate. Random graph was created upon salama [10] graph which was also used in [2][4][6]. Multicast group nodes that are about 30 percent of all nodes of the graph have been selected randomly. Source node, destination node and weights of edge of multicast tree were created randomly. In order to compare the genetic algorithm upon [7] and particle swarm algorithm according [2] were applied.

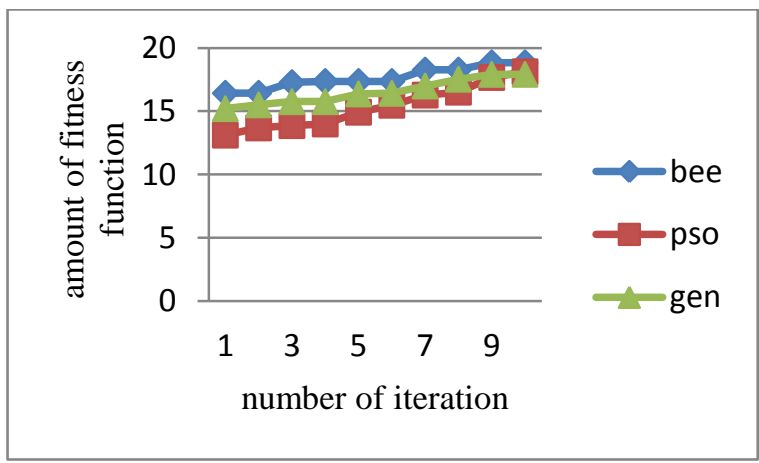

Figure1- diagram of the best amount of fitness function in each repetition.

In the figure, the highest achieved fitness for multicast tree created from the performance of relevant algorithm on network graph was presented in repetition of each algorithm. In this performance the numbers of network nodes were considered 15 and the numbers of trees created from the primary performance of algorithm were considered 10 .

The results don't show being appropriate amount of fitness of proposed algorithm. Bee algorithm considers a number of neighbors and then selects them and because of entering more random trees in issue area has more ability than putting in the best place. Although with the respect that every three algorithms have been made suitable with almost the same primary answer, in every step more suitable results were achieved by bee algorithm.

The average of fitness amounts were achieved during 15 repetitions is 17.62 that all of the data have the amount of 1.2 in deviation criteria and as seen in the figure in last several repetitions the algorithm didn't also find a more appropriate answer.That's why all of these cases are causes to be convergence of the issue with the last amount of achieved fitness.

In another consideration, the amounts of fitness have been considered about increasing the number of nodes in the network.

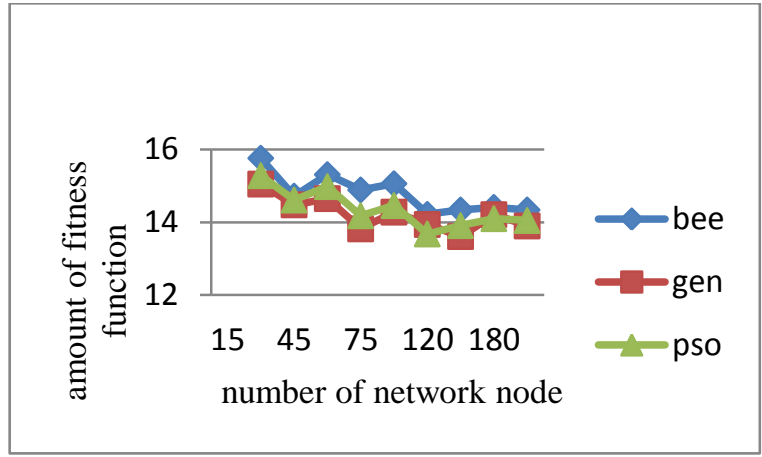

Figure2-diagram of the best fitness function with more nodes.

The results show that when the number of network nodes increases, bee algorithm has more appropriate fitness function amount than genetic algorithm and particle Swarm.

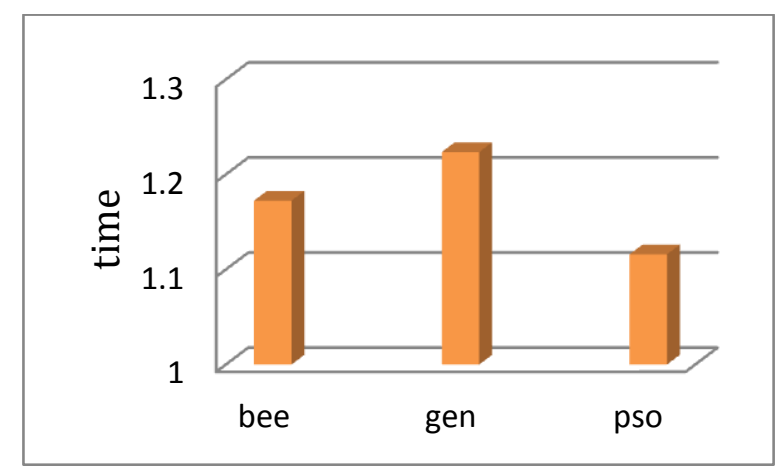

Figure 3-diagram of average tome of 15 times of algorithm performance.

As it can been seen in the figure, the amount of performance time of bee algorithm is better than the genetic algorithms and it is slower than the amounts of performance time of particle Swarm algorithm.

\section{Conclusion}

The proposed algorithm has gotten pattern from bee behavior that is a new work in routing of multicast service quality. With this respect that bee algorithm is an algorithm to solve the issue of optimizing which has the features of getting rid of local searching area and finds and appropriate percent of application in rates of bandwidth, delaying and cost than genetic algorithm and particle Swarm optimization of Steiner tree. 


\section{References}

[1] Hua Wang, Xiangxu Meng, Shuai Li, Hong Xu, Atreebased particle swarm optimization for multicast routing. Computer Networks 54(15): 2775-2786 (2010)

[2] Ziqiang Wang, Xia Sun, Dexian Zhang, "A PSO-Based Multicast Routing Algorithm," icnc, vol. 4, p.664-667, Third International Conference on Natural Computation (ICNC 2007), 2007

[3] Junwei Wang,Zhaoxia Wu, PSO based QoS Multicast Routing Scheme under inaccurate network Information, Intelligent Computing and Intelligent Systems (ICIS), 2010 IEEE International Conference on 568

[4] Hua Wang; Zhao Shi; Jun Ma; Sch. of Comput. Sci. \& Technol., Shandong Univ., Jinan, A Modified Ant Colony Algorithm for Multi-constraint Multicast Routing ,Communications and Networking in China, 2006, 1 - 5

[5] Xi-hong Chen, Shao-wei Liu, Jiao Guan, Qiang Liu, "Study on QoS Multicast Routing Based on ACO-PSO Algorithm," icicta, vol. 3, pp.534-537, International Conference on Intelligent Computation Technology and Automation, 2010
[6] D.T. Pham, A. Ghanbarzadeh, E. Koc, S. Otri, S. Rahim and M.Zaidi," The Bees Algorithm-A novel tool for complex optimization problems". Proceedings of IPROMS 2006 Conference.pp. 151-168, 2006.

[7] Haghighat A. T., Faez K., Dehghan M, Mowlaei A., Ghahremani Y., "GA-Based Heuristic Algorithms for Bandwidth-delay-constrained Least-cost Multicast Routing," Computer Communications Journal (Elsevier Science), Vol. 27, No. 1, PP. 111-127, 2004.

[8]. Pham D T, Ghanbarzadeh A, Koc E, Otri S, Rahim S and Zaidi M, 2005, The Bees Algorithm, Technical Note, Manufacturing Engineering Centre,Cardiff University, UK.

[9] Z. Wang, B. Shi, E. Zhao, Bandwidth-delay-Constrainted least cost multicast routing based on heuristic genetic algorithm' Computer Communications 24(2001) 685692.

[10] H.F.Salama, D.S.Reeves, and Y.Viniotis, "Evaluation of multicast routing algorithm for real-time communication on high-speed networks", IEEE Journal on Selected Areas in Communications, IEEE Press, Los Alamitos, CA, April 1997, pp. 332-345. 